\title{
Gestores, Engajamento e Comportamentos Políticos: Uma Relação Não Linear
}

\author{
Managers, Engagement and Political Behaviors: A Nonlinear Relationship
}

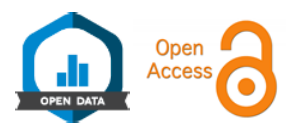

\author{
Romulo Matos de Moraes ${ }^{10}$ \\ Aridelmo José Campanharo Teixeira ${ }^{2}$
}

\section{RESUMO}

Contexto: normalmente ligados aos centros de poder e tomada de decisão, os gestores encontram-se mais próximos aos efeitos da Percepção de Política nas Organizações, um fenômeno considerado contraproducente, porém inerente à própria existência das instituições. Objetivo: este estudo investiga como gestores de diversas empresas, com diferentes níveis de Engajamento no Trabalho encaram a presença de comportamentos políticos em suas organizações. Método: dados de uma survey com 1498 gestores foram submetidos a análise de clusters; regressões múltiplas lineares e não lineares subsidiaram testes de hipóteses. Resultados: na maioria dos casos analisados, a resiliência, o envolvimento e a concentração mitigaram a percepção de comportamentos políticos na organização. Entretanto, constatou-se uma quebra de paradigma entre os altamente engajados: o Engajamento impactou no aumento da Percepção de Política. Conclusões: Engajamento no Trabalho e Percepção de Política na Organização relacionamse de forma curvilínea, indicando que não são necessariamente antagônicos. O estudo indica que gestores emocionalmente e cognitivamente estruturados tendem a aumentar seu engajamento mesmo diante de uma elevada percepção de um ambiente político, revelando uma perspectiva positiva às práticas de gestão: fomentar um maior entendimento e conexão com o ambiente organizacional possivelmente trará resultados mais eficazes do que tentativas de coibir ou negligenciar os comportamentos políticos.

Palavras-chave: engajamento no trabalho; percepção de política na organização; comportamento organizacional; relacionamento não linear.

Classificação JEL: D72, G32, C2.

'Instituto Federal de Educação, Ciência e Tecnologia do Espírito Santo, Alegre, ES, Brasil. ${ }^{2}$ FUCAPE Business School, Vitória, ES, Brasil.

\section{ABSTRACT}

Context: usually linked to power and decision making, managers are much closer to the effects of the Perceptions of Organizational Politics, a counterproductive phenomenon, but inherent in the very existence of institutions. Objective: the purpose of this paper is to investigate how managers from different companies and with different Work Engagement levels face the presence of political behavior in their organizations. Method: we performed cluster analysis with data from 1498 managers; The hypotheses were tested using multiple linear and nonlinear regression analyzes. The methodology includes descriptive statistics and ANOVA. Results: in most cases, resilience, involvement and concentration, mitigates the perception of political behavior in the organizations. However, there was a paradigm shift among the highly engaged: Engagement impacted on the increase of Perceptions of Organizational Politics. Conclusions: work Engagement and Perceptions of Organizational Politics has a curvilinear relationship, indicating that they are not necessarily antagonistic. The study indicates that emotionally and cognitively structured managers tend to increase their engagement even in the face of a heightened perception of a political ambience, revealing a positive perspective on management practices: fostering greater understanding and connection with the organizational environment will possibly yield more effective results than trying to restrain or neglect political behaviors.

Keywords: work engagement; perception of organizational politics; organizational behavior; nonlinear relationship.

Como citar: Moraes, R. M. de, \& Teixeira, A. J. C. (2020). Managers, engagement and political behaviors: A nonlinear relationship. Revista de Administração Contemporânea, 24(3), 218-231. https://doi.org/10.1590/1982-7849rac2020180255

Editor-chefe: Wesley Mendes-Da-Silva (Fundação Getulio Vargas, EAESP, Brasil) Pareceristas: Paula Foroni (Universidade de São Paulo, FEA, Brasil) Silvio Parodi Oliveira Camilo (Universidade do Extremo Sul Catarinense, PPGDS, Brasil) (] Aceite em: 21/08/2019

\# de revisores convidados até a decisão:

\begin{tabular}{|c|c|c|c|c|c|c|c|c|c|}
\hline & 1 & 2 & 3 & 4 & 5 & 6 & 7 & 8 & 9 \\
\hline $1^{a}$ rodada & (x) & (x) & 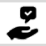 & (x) & $\stackrel{9}{2}$ & & & & \\
\hline $2^{a}$ rodada & (x) & 2 & & & & & & & \\
\hline $3^{a}$ rodada & $\stackrel{9}{2}$ & & & & & & & & \\
\hline $4^{a}$ rodada & 2 & & & & & & & & \\
\hline
\end{tabular}




\section{INTRODUÇÃO}

As organizações pós-modernas são ambientes eminentemente políticos, influenciados por fluxos de mudanças e incertezas em um sistema composto de um lado por atividades em prol de resultados comuns e de outro por uma arena disputada por grupos com diferentes interesses (Brass, 2017; Jackson \& Grace, 2018; Mintzberg, 1985). Gestores de diferentes níveis estão inseridos nesse panorama no qual, das relações de poder envolvidas, decorrem jogos e táticas para influenciar, mudar, obter e garantir interesses inerentes à própria existência das instituições (Kimura, 2015; Mintzberg, 1985).

Nas últimas décadas, a política vem sendo relatada como um fenômeno comum no mundo organizacional e até mesmo necessário à sobrevivência das instituições (Allen, Porter, \& Angle, 2016; Buchanan, 2008; Hochwarter, Ferris, Laird, Treadway, \& Coleman Gallagher, 2010). Ao mesmo tempo, no cotidiano das organizações, os comportamentos relacionados à política são percebidos como atividades não sancionadas, provenientes de manobras de bastidores e ações em interesse próprio. Como consequência, a Percepção de Política na Organização (PPO) é considerada um aspecto ambíguo, estressor e disfuncional do ambiente de trabalho, com impacto na produtividade das equipes (Abbas, Raja, Darr, \& Bouckenooghe, 2014; Bedi \& Schat, 2013; Chang, Rosen, \& Levy, 2009).

Considerando a presença quase inexorável da política na vida das organizações, este estudo, sob a ótica do Comportamento Organizacional Positivo (Luthans, Luthans, \& Luthans, 2015), aborda a necessidade de identificar como fatores positivos relacionam-se com a PPO e assim subsidiar ações gerenciais e pesquisas de Desenvolvimento de Recursos Humanos voltadas a minimizar os aspectos negativos da PPO e ampliar o entendimento de seus meandros. Para esta proposta, será utilizado o conceito Engajamento no Trabalho (EGT): um estado em que os profissionais se conectam a suas atividades laborais através de dimensões energéticas, emocionais e cognitivas (Schaufeli, Bakker, \& Salanova, 2006).

A argumentação aqui desenvolvida não se fixou apenas na relação positivo versus negativo, pois, apesar da predominância de resultados indicando a política percebida como um estressor, a adição de outras abordagens preenche lacunas e amplia as perspectivas de investigação: estudos indicam que, no nível individual, o que se apresenta como obstáculo para alguns, pode ser percebido como oportunidade por outros, com diferentes nuances, resultando em grupos com comportamentos distintos frente a um mesmo fator (LePine, Podsakoff,
\& LePine, 2005; Lu, Wang, Lu, Du, \& Bakker, 2014; Meisler \& Vigoda-Gadot, 2014; Perrewé, Rosen, \& Maslach, 2012). Recentemente, a relação entre EGT e aspectos contraproducentes ainda apresentou resultados inconclusivos (Reijseger, Peeters, Taris, \& Schaufeli, 2017). Da mesma forma, é possível identificar inconsistências quanto ao efeito negativo da PPO junto a fatores laborais (para uma meta-análise, veja Miller, Rutherford, \& Kolodinsky, 2008).

Especificamente quanto ao estudo da política organizacional, a maioria das investigações nesta área ocorreu na Europa e Estados Unidos (Bedi \& Schat, 2013; Rich, LePine, \& Crawford, 2010). Entretanto, em economias emergentes como Brasil, Rússia, Índia e China, identifica-se uma tendência a criar arranjos informais convergentes com os interesses de grupos específicos, e à sobreposição das normas sancionadas (Ardichvili, Jondle, Kowske, Cornachione, Li \& Thakadipuram, 2012). $\mathrm{Na}$ presente pesquisa, o cenário é o Brasil, onde historicamente há um contexto fortemente afetado pelos comportamentos políticos (De Moraes, 2017; Mansur \& Sobral, 2011; Torres, Alfinito, Galvão, \& Tse, 2015).

O objetivo deste artigo é investigar como gestores de diversas empresas, com diferentes níveis de engajamento, encaram a presença de comportamentos políticos em suas organizações. Paraumaprofundamento das análises, a característica multidimensional do EGT foi explorada e a hipótese de relação curvilínea foi considerada.

Em um contexto em que o estudo da força da política no ambiente organizacional ainda é tabu ou abordado de forma secundária (Mansur \& Sobral, 2011; Miranda, 2009), esta pesquisa contribui com uma alternativa à generalização negativa do fenômeno ao conduzir uma abordagem não linear em suas análises e considerar que a política pode ser percebida ora como obstáculo ora como oportunidade. Os resultados encontrados sobre a ação do EGT frente à PPO contribuem também para auxiliar na definição de fronteiras entre fomento e mitigação destes dois fatores. Os achados apresentam uma perspectiva positiva às práticas de gestão, pois apontam que promover uma maior conexão com o ambiente organizacional potencialmente trará resultados mais eficazes do que tentar coibir os comportamentos políticos ou negligenciá-los.

\section{ENGAJAMENTO NO TRABALHO}

Na Academia, EGT é um conceito relativamente novo nas pesquisas sobre gerenciamento de recursos humanos e possui uma abordagem identificada no campo do Comportamento Organizacional Positivo, 
no qual são destacados os aspectos edificantes e positivos da personalidade humana, buscando-se assim a manutenção de um funcionamento ótimo do indivíduo e, por consequência, um gerenciamento organizacional sob esse prisma (Gagné, 2014; Luthans et al., 2015; Schaufeli, 2012).

Trata-se de um estado mental composto por três dimensões: Vigor, Absorção e Dedicação. A dimensão Vigor é caracterizada por elevados níveis de energia mental, forte resiliência durante o trabalho e uma persistente vontade de investir esforço nas tarefas, mesmo em situações adversas, o que permite ao indivíduo lidar com problemas, superar obstáculos ou resistir à pressão cotidiana (Gagné, 2014; Schaufeli, 2012; Schaufeli, Salanova, González-Romá, \& Bakker, 2002). O fator emocional Dedicação se refere a estar fortemente envolvido em sua tarefa, experimentando assim uma sensação de significância, entusiasmo, inspiração, orgulho e desafio (Gagné, 2014; Schaufeli et al., 2002). Por fim, a dimensão cognitiva Absorção é caracterizada por um estado em que se está totalmente concentrado e prazerosamente absorto em seu trabalho (Gagné, 2014; Schaufeli et al., 2002). Nessa condição, o grau de absorção faz com que o indivíduo experimente a sensação de que o tempo passa depressa, de forma fluida, e não consiga facilmente desligar-se do trabalho (Schaufeli et al., 2002; Schaufeli, 2012).

Implicações importantes do EGT estão ligadas ao alto nível de comprometimento, satisfação no trabalho, baixo absenteísmo e forte motivação para aprender (Bakker \& Demerouti, 2008, 2017; Rich et al., 2010). Todas as características descritas anteriormente permitem inferir que por sua natureza, indivíduos engajados possuem emoções positivas, energia e recursos pessoais suficientes para enfrentar a tensão e o estresse do dia-a-dia, o que os permite minimizar seus problemas ou encarar como desafio o que costumeiramente é considerado um obstáculo (Bakker \& Demerouti, 2008; Crawford, LePine, \& Rich, 2010; De Moraes \& Teixeira, 2017).

\section{PERCEPCÃO DE POLÍTICA NA ORGANIZAÇÃO}

Este estudo aborda a PPO sob a seguinte perspectiva: percepção de ações cujo resultado trará benefício próprio ou para um grupo e que, sob o aspecto social e organizacional, são consideradas ilegítimas principalmente por não considerar as resultantes negativas para as outras partes (Lepisto \& Pratt, 2012; Mintzberg, 1985).

Em uma pesquisa seminal, Madison, Allen, Porter, Renwick e Mayes (1980) apontaram que gerentes percebiam a atividade política relacionada ao poder e à incerteza, porém também a consideravam como uma questão importante para as organizações e para os indivíduos. A presença da política como um sistema de influência inerente aos diversos tipos de instituição é recorrentemente investigada sob a ótica da percepção disfuncional que os funcionários têm do fenômeno e seus impactos negativos (Allen et al., 2016; Bedi \& Schat, 2013; Miller et al., 2008). Quanto às resultantes da PPO, pode-se destacar: forte relação negativa com satisfação no trabalho e comprometimento afetivo; ef eito restritivo no desempenho de tarefas; redução na dedicação dos funcionários (Chang et al., 2009; Mansur \& Sobral, 2011; Miller et al., 2008).

No contexto brasileiro, há indicativos de que a presença e os efeitos da política organizacional são conhecidos e praticados em diversos níveis das organizações, já que os valores culturais do país em muitos casos fortalecem a posição dos indivíduos a partir da política e, ao mesmo tempo, amenizam sua caracterização como um fenômeno negativo (Chu \& Wood, 2008; Torres et al., 2015), permitindo que gestores envolvidos em atividades políticas institucionalmente sancionadas propiciem vantagens aos grupos com os quais se relacionam e à organização como um todo (Fedor, Maslyn, Farmer, \& Bettenhausen, 2008).

Entretanto, a política pode ser percebida de diferentes formas: em decorrência de variações emocionais ou cognitivas, em função da forma como se encara o que é oportunidade ou obstáculo, ou ainda por diferenças culturais (Meisler \& VigodaGadot, 2014; Perrewé et al., 2012; Vigoda, 2001).

Os gestores desempenham um papel-chave nas mudanças organizacionais e possuem uma ligação muito estreita com o poder e suas táticas (Buchanan, 2008; Hope, 2010). A busca por posições hierárquicas impulsiona atitudes voltadas à aproximação com os centros de poder e controle, onde ocorrem processos de tomada de decisão, normalmente suscetíveis à influência política (Allen et al., 2016; Atinc, Darrat, Fuller, \& Parker, 2010). Poucas decisões importantes são tomadas sem que as partes-chave protejam ou melhorem seus interesses (Kreutzer, Walter, \& Cardinal, 2015). Disto decorre a percepção de que a dimensão política é um fenômeno existente nos níveis gerenciais ou fortemente associado àqueles que buscam galgar posições nesta direção, independente dos meios utilizados (Allen et al., 2016; Chang et al., 2009).

Como se pode ver, o desafio em captar a percepção do comportamento político nas organizações está ligado à ambiguidade, subjetividade e controvérsia do assunto (Allen et al., 2016; Miller et al., 2008). Dependendo das experiências prévias de cada observador, metas pessoais de carreira, gênero e grupo do qual faz parte, um mesmo comportamento pode ser considerado político ou não (Ferris, Frink, Bhawuk, 
Zhou, \& Gilmore, 1996; Kacmar, Bachrach, Harris, \& Zivnuska, 2011; Kacmar, Bozeman, Carlson, \& Anthony, 1999) ou ainda ser encarado como um viabilizador dos objetivos organizacionais e um mecanismo de proteção de interesses específicos (Russo, Rodrigues, Russo, \& Yu, 2018).

\section{FORMULAÇÃO DE HIPÓTESES}

Como revisado anteriormente, apesar de predominantes nas pesquisas, os vínculos negativos entre PPO e fatores produtivos não são um consenso. Assim, o desenvolvimento deste estudo considera que a associação de um estado positivo com uma percepção negativa pode gerar uma combinação de complexidade muito menos linear e generalizada que as tradicionais pesquisas organizacionais (Warr, 2011). Sob esta ótica não linear e considerando a importância da variabilidade nos níveis de análise ao se tratar de características complexas (Nishii \& Wright, 2008; De Moraes \& Teixeira, 2017), as hipóteses foram segmentadas em grupos com níveis baixos, intermediários e elevados de EGT, conforme descrito a seguir.

Utilizou-se primeiramente as dimensões do EGT no teste de hipóteses, o que permitiu um maior aprofundamento sobre os fatores estruturantes deste conceito frente à variável dependente (PPO), evitando-se assim perda de informação (Seppälä et al., 2009). Essa prática visa fornecer subsídios para que as análises posteriores utilizem o EGT consolidado de forma fundamentada quanto à não linearidade (Schaufeli et al., 2006; Seppälä et al., 2009).

Os dois primeiros grupos de hipóteses adotam a perspectiva do Comportamento Organizacional Positivo, no qual o foco são os valores positivos e não a tradicional abordagem voltada ao negativo/ problemático (Luthans et al., 2015). Já que funcionários engajados têm atributos suficientes para concentrar esforços em suas atividades e reduzir o efeito das adversidades (Rich et al., 2010; Schaufeli, 2012), pode-se esperar um efeito mitigador do EGT frente à PPO, permitindo assim uma associação negativa entre estes atributos e a PPO:

Hipótese 1: As dimensões VIGOR $\left(\mathrm{H}_{1 \mathrm{a}}\right)$, DEDICAÇÃO $\left(\mathbf{H}_{\mathbf{1 b}}\right)$ e ABSORÇÃO $\left(\mathbf{H}_{1 \mathrm{c}}\right)$ estão negativamente relacionadas à PPO em um grupo com baixo escore de EGT.

Como extensão da argumentação anterior, e considerando que o estoque de recursos dos engajados possui um efeito acumulativo na capacidade de responder a obstáculos recorrentes (Gagné, 2014; Reijseger et al., 2017), pode-se ainda propor que, com um aumento no nível de EGT, a força da relação negativa entre suas dimensões e a PPO seja maior:

Hipótese 2: No grupo com escore intermediário de EGT, a relação negativa entre as dimensões VIGOR $\left(\mathbf{H}_{2 \mathbf{a}}\right)$, DEDICAÇÃO $\left(\mathbf{H}_{\mathbf{2 b}}\right)$, ABSORÇÃO $\left(\mathbf{H}_{2 \mathrm{c}}\right)$ e PPO tem maior magnitude em relação ao grupo de baixo escore de EGT.

Entretanto, funcionários altamente engajados dispõem de condições energéticas, cognitivas e emocionais suficientemente sólidas para desenvolver forte conexão e entendimento do ambiente em que se encontram (Bakker \& Demerouti, 2008; Christian, Garza, \& Slaughter, 2011), o que permite uma maior percepção de estressores, sem prejuízo para as características positivas que trazem consigo, uma vez que tratam obstáculos como oportunidades (Crawford et al., 2010; De Moraes \& Teixeira, 2017; Kane-Frieder, Hochwarter, \& Ferris, 2014). Essa linha argumentativa pode ser traduzida em relações positivas entre as dimensões do EGT e a PPO:

Hipótese 3: No grupo com escore elevado de EGT, a relação entre as dimensões VIGOR $\left(\mathbf{H}_{3 \mathbf{a}}\right)$, DEDICAÇÃO $\left(\mathbf{H}_{3 \mathrm{~b}}\right)$, ABSORÇÃO $\left(\mathbf{H}_{3 \mathrm{c}}\right)$ e a PPO é positiva.

Por fim, ao se contrapor H1 e H2 com H3, é possível identificar que a literatura sustenta dois caminhos: de um lado uma tradicional associação negativa entre fatores produtivos e estressores (Bedi \& Schat, 2013; Chang et al., 2009; Miller et al., 2008) e de outro a capacidade que os altamente engajados possuem para tratar tais adversidades, resultando, de forma inversa, em uma associação positiva (Crawford et al., 2010; Kane-Frieder et al., 2014). Esta contradição deixa uma lacuna sobre o poder de explicação das abordagens lineares ao se confrontar conceitos de Comportamento Organizacional. Diante disso, levanta-se a hipótese de que um modelo curvilíneo possa ser mais apropriado para demonstrar a relação geral entre os construtos EGT e PPO:

Hipótese 4: EGT e PPO apresentam uma relação curvilínea quadrática positiva $\left(\mathbf{H}_{4}\right)$.

\section{MÉTODO}

\section{Amostra e procedimento}

A amostra é representada por Administradores registrados em um Conselho Regional de Administração (CRA) de um estado brasileiro. A coleta de dados contou com apoio do CRA que encaminhou institucionalmente a 9351 administradores registrados um convite 
para participar da pesquisa. Em relação ao total de administradores brasileiros registrados nos Conselhos, o CRA estudado representa $4,23 \%$. No site da instituição um link ficou disponível para acessar o questionário eletrônico. Ao longo de três meses, em três boletins de notícias do CRA, o convite foi reiterado. Foram obtidas 1498 respostas válidas para o estudo. A opção por pesquisar apenas administradores e afiliados ao CRA se deu por dois motivos: primeiramente por ser uma área de formação que, por sua natureza, possivelmente teria respondentes atuando em algum nível de gestão, objeto deste estudo; o segundo critério foi a acessibilidade propiciada pelo apoio do CRA, o que ampliou potencialmente a coleta, permitindo acesso direto a profissionais de diversas empresas. Utilizaram-se apenas os dados de respondentes do setor privado e que se autodeclararam estar posicionados em algum nível hierárquico de gestão. O tempo médio de atuação na empresa foi 7 anos; todos possuem graduação e destes $54,2 \%$ possuem algum tipo de pós-graduação; a idade média foi 34 anos. A maioria atua no setor de serviços, em empresas de pequeno e médio porte nas áreas de administração geral, finanças, comercial e recursos humanos. A Tabela 1 apresenta os demais dados da caracterização da amostra.

Tabela 1. Perfil dos respondentes.

\begin{tabular}{|c|c|c|c|}
\hline Característica & Subdivisão & Quantidade & Percentual \\
\hline \multirow{4}{*}{ Tempo de empresa } & Até 5 anos & 503 & $33,6 \%$ \\
\hline & 6 a 10 anos & 827 & $55,2 \%$ \\
\hline & 11 a 15 anos & 116 & $7,7 \%$ \\
\hline & Acima de 15 anos & 52 & $3,5 \%$ \\
\hline \multirow{4}{*}{ Escolaridade } & Somente graduação & 686 & $45,8 \%$ \\
\hline & Especialização & 755 & $50,4 \%$ \\
\hline & Mestrado & 49 & $3,3 \%$ \\
\hline & Doutorado & 8 & $0,5 \%$ \\
\hline \multirow{2}{*}{ Gênero } & Feminino & 474 & $31,6 \%$ \\
\hline & Masculino & 1024 & $68,4 \%$ \\
\hline \multirow{4}{*}{ Idade } & Até 30 anos & 539 & $36,0 \%$ \\
\hline & de 31 a 40 anos & 698 & $46,6 \%$ \\
\hline & de 41 a 50 anos & 204 & $13,6 \%$ \\
\hline & Acima de 50 anos & 57 & $3,8 \%$ \\
\hline \multirow{4}{*}{ Porte da empresa } & Microempresa & 328 & $21,9 \%$ \\
\hline & Pequeno & 460 & $30,7 \%$ \\
\hline & Médio & 554 & $37,0 \%$ \\
\hline & Grande & 156 & $10,4 \%$ \\
\hline \multirow{3}{*}{ Setor econômico } & Primário (ex.: agricultura, pecuária, extrativismo) & 66 & $4,4 \%$ \\
\hline & Secundário (ex.: indústria, construção civil) & 410 & $27,4 \%$ \\
\hline & Terciário (ex.: serviços, comércio) & 1022 & $68,2 \%$ \\
\hline \multirow{4}{*}{ Área de atuação } & Administração geral & 886 & $59,1 \%$ \\
\hline & Finanças & 181 & $12,1 \%$ \\
\hline & Comercial & 316 & $21,1 \%$ \\
\hline & Recursos Humanos & 115 & $7,7 \%$ \\
\hline \multirow{3}{*}{ Nível hierárquico na gestão } & Inicial & 532 & $35,5 \%$ \\
\hline & Intermediário & 520 & $34,7 \%$ \\
\hline & Alto & 446 & $29,8 \%$ \\
\hline
\end{tabular}

Nota. Essa tabela apresenta o perfil dos gestores que participaram do estudo. A maioria dos respondentes é do gênero masculino, com até 40 anos de idade, mais de 5 anos de tempo de serviço na empresa, atuam predominantemente em organizações de pequeno e médio porte e no setor terciário. Fonte: Dados da Pesquisa. 


\section{Questões éticas}

Os procedimentos para a realização deste estudo foram analisados e aprovados pelo Comitê de Ética em Pesquisa com Seres Humanos do Ifes (CEP/Ifes), ligado à Comissão Nacional de Ética em Pesquisa do Ministério da Saúde do Brasil - CONEP/MS (Brasil, 2017). O projeto deste estudo encontra-se cadastrado no CONEP/MS sob o CAAE (Certificado de Apresentação de Apreciação Ética) número 47091015.1.0000.5072 de 16/09/2015. O comitê apontou que na pesquisa os interesses dos participantes foram respeitados em sua integridade e dignidade e que a proposta se encontra dentro de padrões éticos científicos.

Sobre este processo, pode-se destacar que foi garantido aos respondentes: que se tratava de uma participação voluntária, que as respostas seriam anônimas e confidenciais e que o tratamento dos dados ocorreria de forma agregada. Os participantes tiveram acesso a um Termo de Consentimento Livre e Esclarecido (TCLE) com as informações necessárias para decidir ou não pela participação.

\section{Medidas}

Engajamento no trabalho (EGT): Utilizou-se a versão de nove itens da Utrecht Work Engagement Scale (UWES-9) com 3 itens para cada dimensão do conceito (Schaufeli et al., 2006), validada para o português por Agnst, Benevies-Pereira e Porto-Martins (2009). Tratase de uma escala de sete pontos variando de 1 (Nunca/ Nenhuma vez) a 7 (Sempre/todos os dias). Exemplos de itens são: Em meu trabalho, sinto-me repleto de energia; e Estou orgulhoso com o trabalho que realizo. Neste estudo os coeficientes de consistência interna foram 0,868 para vigor, 0,897 para dedicação, 0,904 para absorção e 0,886 para EGT.

Percepção de Política na Organização (PPO): Utilizou-se a Escala de Percepção de Política na Organização para o Brasil (EPPO-Br), com 6 itens (De Moraes, 2017). O formato de resposta foi uma escala de concordância de 5 pontos, variando de 1 (Discordo totalmente) a 5 (Concordo totalmente). Exemplos de Itens: Muitos funcionários fazem manobras para se inserir em grupos; e As pessoas estão trabalhando nos bastidores a fim de garantirem seu espaço. A escala apresentou uma boa confiabilidade de coerência interna $(\alpha=0,812)$.

Variáveis de controle: nível hierárquico como gestor (1-inicial; 2-intermediário; 3-alto), gênero (1-feminino; 2-masculino) e tempo de empresa (em anos). Julgou-se necessário incluí-las nas regressões dadas as possíveis covariações com as variáveis focadas neste estudo. Além disso, pesquisas prévias sugerem esta possibilidade (Kacmar et al., 1999; Kane-Frieder et al., 2014; Schaufeli, 2012).

\section{Estratégia de análise}

Inicialmente, para realizar a análise entre os grupos com escores baixos, médios e altos de EGT foi necessário identificar, em relação à amostra, a existência de grupos com tais características. Optou-se por uma análise de clusters em duas etapas (agrupamento sequencial seguido por método hierárquico), mais indicada para amostras de maior escala (Garson, 2014). Nesse processo foram incluídas as variáveis latentes do estudo para identificar agrupamentos naturais e reduzir o erro da variabilidade combinada. A significância estatística entre as diferenças das médias dos grupos foi verificada por uma One-way ANOVA. Para um maior detalhamento desta abordagem, sugere-se o trabalho de Sarsted e Mooi (2014).

Para testar $\mathbf{H}_{1 \mathbf{a}, \mathbf{b}, \mathbf{c}}, \mathbf{H}_{2 \mathbf{a}, \mathbf{b}, \mathbf{c}}$ e $\mathbf{H}_{3 \mathbf{a}, \mathbf{b}, \mathbf{c}}$, buscou-se identificar a magnitude e o tipo de associação entre as dimensões do EGT (variáveis independentes) e PPO (variável dependente). Regressões múltiplas foram conduzidas em cada um dos grupos resultantes da análise de clusters. A equação (1) considera as variáveis independentes VIGOR, DEDICAÇÃO e ABSORÇÃO e as variáveis de controle NÍVEL HIERÁRQUICO, GÊNERO e TEMPO de EMPRESA. A variável dependente $Y_{P P O}$ representa a Percepção de Política no grupo analisado; o termo $\varepsilon$ representa o erro experimental e $\beta_{0}$ a constante de regressão.

Para o teste de $\mathbf{H}_{4}$, também foram consideradas as variáveis de controle. Uma regressão hierárquica foi aplicada à amostra total tendo como variáveis independentes EGT (escore consolidado) na primeira etapa e o componente quadrático $\left(\mathrm{EGT}^{2}\right)$ na segunda etapa, conforme o modelo apresentado na equação (2). Para um maior detalhamento desses procedimentos sugere-se Cohen, West e Aiken (2014).

$$
\begin{aligned}
& Y_{P P O}=\beta_{0}+\beta_{I} \text { NIIVELHIERÁRQUICO }+\beta_{2} \text { GÊNERO }+ \\
& \beta_{3} \text { TEMPOEMPRESA }+\beta_{4} \text { VIGOR }+\beta_{5} \text { DEDICAÇÃO }+ \\
& \beta_{6} \text { ABSORÇ } \tilde{O}+\varepsilon . \\
& Y_{P P O}=\beta_{0}+\beta_{1} \text { NÍVELHIERÁRQUICO }+\beta_{2} \text { GÊNERO }+ \\
& \beta_{3} \text { TEMPOEMPRESA }+\beta_{4} E G T+\beta_{5} E G T^{2}+\varepsilon .
\end{aligned}
$$

\section{ANÁLISE E DISCUSSÃO DOS RESULTADOS}

A Tabela 2 demonstra os desvios-padrão (DP) e resultados médios $(\mathrm{M})$ das variáveis latentes segundo os agrupamentos resultantes da análise de clusters, bem como da amostra total. Pode-se perceber pela estatística F da comparação entre grupos que as diferenças nas médias dos conglomerados são altamente significativas $(\mathrm{p}<0.001)$. 
Tabela 2. One-way ANOVA e estatísticas descritivas.

\begin{tabular}{|c|c|c|c|c|c|c|c|c|c|c|}
\hline \multirow{3}{*}{$\begin{array}{l}\text { Variáveis } \\
\text { latentes }\end{array}$} & \multicolumn{2}{|c|}{ Amostra total } & \multicolumn{2}{|c|}{ ANOVA } & \multicolumn{2}{|c|}{$\begin{array}{l}\text { EGT Baixo } \\
\text { (Cluster 1) }\end{array}$} & \multicolumn{2}{|c|}{$\begin{array}{l}\text { EGT Médio } \\
\text { (Cluster 2) }\end{array}$} & \multicolumn{2}{|c|}{$\begin{array}{l}\text { EGT Alto } \\
\text { (Cluster 3) }\end{array}$} \\
\hline & \multicolumn{2}{|c|}{$\mathrm{N}=1498$} & \multicolumn{2}{|c|}{$\begin{array}{c}\text { Comparação entre } \\
\text { grupos }\end{array}$} & \multicolumn{2}{|c|}{$\mathrm{N}=409$} & \multicolumn{2}{|c|}{$\mathrm{N}=791$} & \multicolumn{2}{|c|}{$\mathrm{N}=298$} \\
\hline & M & DP & & & M & DP & M & DP & M & DP \\
\hline VIGOR & 4,42 & 1,58 & 1564,63 & $* * *$ & 2,62 & 0,93 & 4,56 & 1,01 & 6,26 & 0,56 \\
\hline DEDICAÇÃO & 4,63 & 1,73 & 1947,91 & $* * *$ & 2,53 & 1,05 & 4,87 & 0,98 & 6,58 & 0,43 \\
\hline ABSORÇÃO & 4,50 & 1,72 & 1788,77 & $* * *$ & 2,44 & 0,90 & 4,75 & 1,10 & 6,39 & 0,50 \\
\hline EGT & 4,52 & 1,51 & 4808,96 & $* * *$ & 2,53 & 0,63 & 4,73 & 0,58 & 6,41 & 0,36 \\
\hline PPO & 3,82 & 0,78 & 677,12 & $* * *$ & 4,26 & 0,62 & 3,23 & 0,63 & 4,41 & 0,40 \\
\hline
\end{tabular}

Nota. Essa tabela apresenta a estatística descritiva dos dados segmentados pela análise de clusters. A clusterização apresenta três grupos bem distintos quanto ao nível de EGT. Internamente esses grupos são homogêneos quanto à PPO e o EGT (baixo desvio-padrão), propiciando para as análises posteriores uma redução no erro da variabilidade combinada.PPO (Percepção de Política na Organização). EGT: Engajamento no Trabalho.

***: Significante ao nível de $1 \%$. Fonte: dados da pesquisa.

O processo de aglomeração identificou blocos com características bem distintas quanto às médias de Engajamento: O cluster 1 representa 27,30\% da amostra, podendo-se destacar a variável EGT com uma média $(\mathrm{M}=2,53)$ bem abaixo do ponto médio da escala (4) e abaixo ainda da média amostral $(\mathrm{M}=4,52)$. No outro extremo, o cluster 3 , com 19,89\% da amostra, apresentou um EGT médio muito elevado $(M=6,41)$, bem próximo do limite superior da escala (7), e um desvio padrão consideravelmente baixo ( $\mathrm{DP}=0,36$ ) indicando que neste grupo os dados apresentam homogeneidade quanto a este construto. Com o maior número de indivíduos (52,80\% da amostra), o cluster 2 reúne características representativas de uma posição de maior normalidade na qual a média indica respondentes regularmente engajados, tendendo para frequentemente $(\mathrm{DP}=0,58 \mathrm{e}$ $M=4,73)$. A diferença entre o desvio-padrão do EGT da amostra total ( $\mathrm{DP}=1,51)$ e dos clusters $(0,63 ; 0,58 ; 0,36)$, aliada à grande diferença de médias, possibilita acessar níveis críticos de engajamento, conforme proposto por Bakker, Albrecht e Leiter (2011).

Nos clusters 2 e 3 a variável DEDICAÇÃO obteve médias superiores às outras dimensões do EGT, sinalizando que o fator emocional tem um peso relevante na construção do engajamento nestes grupos. Este achado é consistente com propostas que situam as emoções como base para o desenvolvimento de fatores intelectuais e psicológicos, necessários para responder às demandas do ambiente (Meisler \& Vigoda-Gadot, 2014; Smollan, 2014).

A amostra total e os agrupamentos apresentaram uma PPO alta, considerando-se que o ponto máximo da escala é cinco, entretanto estes escores da iniciativa privada foram menores que os encontrados recentemente para o setor público brasileiro (De Moraes, 2017; De Moraes \& Teixeira, 2017). Os desvios-padrão da PPO apurados no conjunto de dados e nos três grupos $(\mathrm{DP}=0,78 ; \mathrm{DP}=0,62 ; \mathrm{DP}=0,63$ e $\mathrm{DP}=0,40)$ indicam um consenso reforçando estudos anteriores que tratam a política como um fator inerente às instituições (KaneFrieder et al., 2014; Parker, Dipboye, \& Jackson, 1995) e sustentando empiricamente a noção de que no caso brasileiro o fenômeno é altamente disseminado (Chu \& Wood, 2008; Torres et al., 2015).

Quanto às regressões múltiplas, seus pressupostos e testes de robustez foram aplicados para cada um dos clusters analisados, uma vez que os três primeiros grupos de hipóteses também foram apresentados de forma segmentada, conforme exposto na Tabela 3:

Tabela 3. Análises dos modelos de regressão.

\begin{tabular}{|c|c|c|c|c|c|c|}
\hline Clusters & $\begin{array}{c}\mathrm{R}^{2} \\
\text { Ajustado } \\
\end{array}$ & VIF & $\begin{array}{l}\text { Independência dos } \\
\text { resíduos: } \\
\text { Durbin-Watson } \\
\end{array}$ & Anova (F) & $\begin{array}{l}\text { Influência de outliers: } \\
\text { Distância de Cook }\left(\mathrm{D}_{i}\right)\end{array}$ & $\begin{array}{c}\text { Heterocedasticidade: } \\
\text { Breusch-Pagan (BP) }\end{array}$ \\
\hline 1. EGT Baixo & $30,80 \%$ & $\leq 1,24$ & 1,785 & $33,28 * * *$ & $\leq 0,052$ & $1,124 \mathrm{~ns}$ \\
\hline 2. EGT Médio & $30,70 \%$ & $\leq 1,28$ & 2,043 & $50,99 * * *$ & $\leq 0,023$ & $5,906 \mathrm{~ns}$ \\
\hline 3. EGT Alto & $32,60 \%$ & $\leq 2,31$ & 1,962 & $21,53 * * *$ & $\leq 0,078$ & $0,777 \mathrm{~ns}$ \\
\hline
\end{tabular}

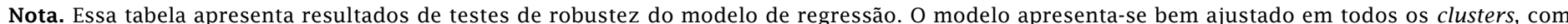

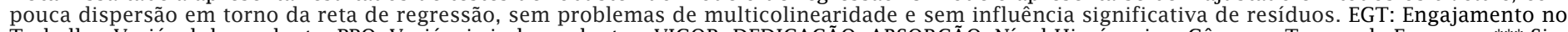

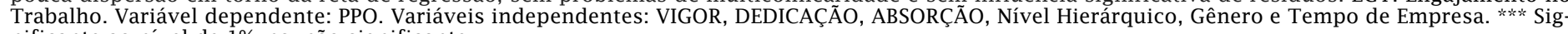
nificante ao nível de $1 \%$. ns: não significante. 
Nos três grupos os coeficientes de determinação $\left(\mathrm{R}^{2}\right)$ ajustados indicam uma boa adequação do modelo, uma vez que é reconhecida a existência de outros antecedentes da PPO, como por exemplo, centralização, hierarquia e ausência de regras (Allen et al., 2016; Kacmar et al., 1999). Os fatores de inflação da variância (VIF) ficaram abaixo de 5, indicando não haver problemas de multicolinearidade entre as variáveis independentes (Hair, Black, Babin, Anderson, \& Tatham, 2006). Com a estatística Durbin-Watson próxima a 2 em todos os clusters, verificou-se que os erros de observações adjacentes não são correlacionados (Hair et al.,
2006). Para todos os casos a Anova foi altamente significante, não foram detectados problemas de heterocedasticidade (BP não significante) e, quanto aos resíduos, não houve influência significativa de outliers $\left(\mathrm{D}_{i}<1\right)$ no conjunto de variáveis preditoras (Chatterjee \& Hadi, 2015; Kaufman, 2013). A solidez dos dados apresentados na Tabela 3 permite conduzir as análises das regressões que suportam os testes de hipóteses a partir dos coeficientes padronizados ( $\beta$ ) estimados nas regressões multivariadas, descritas na Tabela 4:

Tabela 4. Coeficientes das regressões multivariadas.

Níveis de engajamento no trabalho (EGT)

\begin{tabular}{|c|c|c|c|c|c|c|}
\hline \multirow{3}{*}{$\begin{array}{c}\text { Variáveis } \\
\text { independentes }\end{array}$} & \multicolumn{6}{|c|}{ Níveis de engajamento no trabalho (EGT) } \\
\hline & \multicolumn{2}{|c|}{ EGT Baixo (Cluster 1) } & \multicolumn{2}{|c|}{ EGT Médio (Cluster 2) } & \multicolumn{2}{|c|}{ EGT Alto (Cluster 3) } \\
\hline & $\boldsymbol{\beta}$ & $\mathrm{t}$ & $\beta$ & $\mathrm{t}$ & $\beta$ & $\mathrm{t}$ \\
\hline \multicolumn{7}{|l|}{$\begin{array}{l}\text { Variáveis } \\
\text { de interesse } \\
\text { (dimensões): }\end{array}$} \\
\hline VIGOR & $-0,25^{* * *}(\mathrm{H} 1 \mathrm{a})$ & $-6,76$ & $-0,32 * * *(\mathbf{H} 2 \mathrm{a})$ & $-10,32$ & $0,07(\mathbf{H 3 a})$ & 1,21 \\
\hline DEDICAÇÃO & $-0,27 * * *(\mathrm{H} 1 \mathbf{b})$ & $-6,59$ & $0,10 * *(\mathbf{H} 2 \mathbf{b})$ & 2,84 & $0,48 * * *(\mathrm{H} 3 \mathrm{~b})$ & 6,57 \\
\hline ABSORÇÃO & $-0,25 * * *(\mathrm{H} 1 \mathrm{c})$ & $-6,18$ & $-0,37 * * *(\mathrm{H} 2 \mathrm{c})$ & $-12,2$ & $0,21 * * *(\mathrm{H} 3 \mathrm{c})$ & 3,82 \\
\hline
\end{tabular}

\section{Variáveis de controle:}

\begin{tabular}{lcccccc} 
Nível hierárquico & $-0,01$ & $-0,06$ & 0,03 & 1,16 & $0,12^{* *}$ & 1,98 \\
Gênero & 0,06 & 3,37 & 0,05 & 2,13 & 0,06 & 1,24 \\
Tempo de empresa & $0,04^{*}$ & 1,38 & $-0,08$ & $-2,71$ & $-0,01$ & $-0,10$ \\
\hline
\end{tabular}

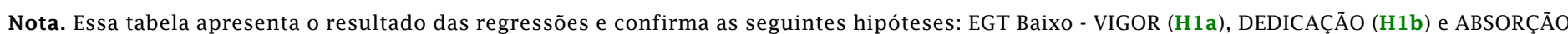

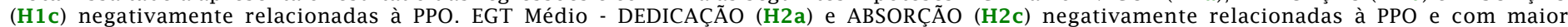

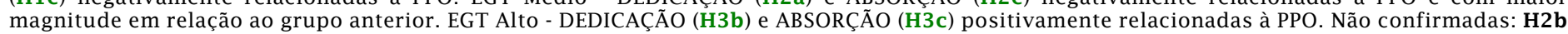
e H3a. Variável dependente: PPO.

*** Significante ao nível de 1\%; ** Significante ao nível de 5\%; * Significante ao nível de $10 \%$.

Considerando-se os níveis de significância e os valores negativos dos coeficientes beta para a regressão do cluster 1 (EGT Baixo), identifica-se que as primeiras hipóteses $\left(\mathbf{H}_{\mathbf{1 a}, \mathbf{b}, \mathbf{c}}\right)$ foram suportadas. As três variáveis independentes se equipararam quanto à importância relativa na previsão da PPO. As associações negativas apresentadas confirmam, no setor privado, a força mitigadora das dimensões do EGT frente à PPO, também identificada no setor público (De Moraes \& Teixeira, 2017), assim como a capacidade dos engajados para enfrentar adversidades (Schaufeli, 2012), reforçando a frequente associação negativa entre fatores produtivos e PPO (Bedi \& Schat, 2013; Chang et al., 2009; Miller et al., 2008).

No grupo com EGT médio, a influência relativa das variáveis VIGOR $(\beta=-0,32)$ e ABSORÇÃO $(\beta=-0,37)$ são maiores que no cluster $1 \quad(\beta=-0,25$ e $\beta=-0,25$, respectivamente), suportando $\mathbf{H}_{2 \mathbf{a}}$ e $\mathbf{H}_{2 \mathrm{c}}$. Assim, no maior agrupamento do estudo, com a menor percepção de política, há uma indicação, com alto nível de significância, de que a resiliência e a concentração dos engajados impactam significantemente na redução da PPO. Entretanto $\mathbf{H}_{\mathbf{2 b}}$ não foi confirmada em função de 
um beta significante, porém positivo para a variável DEDICAÇÃO. Assim, o componente emocional deixa de impactar na redução de PPO entre os funcionários do nível intermediário de EGT. Trata-se de uma alteração relevante no que concerne à transição da interpretação que os indivíduos têm da política como um obstáculo e, além disto, um indicativo da inversão que ocorrerá no próximo cluster.

$\mathrm{Na}$ estimação do grupo de EGT alto, os betas foram positivos, mas apenas as variáveis DEDICAÇÃO e ABSORÇÃO apresentaram resultados estatisticamente significativos, suportando $\mathbf{H}_{3 \mathbf{b}}$ e $\mathbf{H}_{3 \mathbf{c}}$. O resultado desse aglomerado fornece evidências de que há uma ruptura na relação negativa entre EGT e PPO quando se trata de grupos de gestores com alto nível de engajamento em suas atividades. Nesse agrupamento todas as dimensões do EGT e a PPO apresentaram médias altas (Tabela 2) o que em conjunto com o resultado das regressões, apoia estudos como os de Crawford, LePine e Rich (2010) e Kane-Frieder, Hochwarter e Ferris (2014) que indicaram que funcionários altamente engajados percebem estressores de forma positiva, convertendo tal fato em desafio ou oportunidade.

Sobre a associação positiva entre DEDICAÇÃO e PPO presente no cluster 3, é importante destacar que esta variável independente é o fator emocional do EGT, imbuído dos sensos de entusiasmo, orgulho e desafio (Gagné, 2014; Schaufeli et al., 2002). Argumenta-se que no nível interpessoal as emoções são utilizadas estrategicamente como mecanismo de influência e no nível de grupo como fomento de coalisões, com indicações de que alguns indivíduos que genuinamente gostam do jogo político valem-se destas práticas (Allen et al., 2016; Meisler \& VigodaGadot, 2014). Quanto à relação positiva apresentada pelo aspecto cognitivo (ABSORÇÃO), argumentase que os gestores podem considerar a PPO como uma exigência desafiante de suas organizações, desenvolvendo mecanismos de aproximação e aceitação da política organizacional. Esse desafio pode gerar uma estratégia de defesa: ao perceber uma ameaça ao seu bem-estar, o funcionário se engaja em controlar ou alterar a situação (LePine et al., 2005; Perrewé et al., 2012).

Pode-se ainda destacar que no agrupamento com alto EGT houve uma relação positiva entre a variável de controle Nível Hierárquico e a PPO, fornecendo indícios de que com o aumento da posição hierárquica e, portanto, da proximidade com os centros de poder, a percepção de política é ampliada, sem necessariamente prejudicar o engajamento.

Gestores que possuem um alto grau de entendimento e controle sobre o processo político são suscetíveis a ver a política como uma oportunidade de crescimento, esta característica encontra-se presente em outros estressores provenientes do ambiente de trabalho como a ambiguidade e a insegurança (Eldor, 2017; Lu et al., 2014; Perrewé et al., 2012). Nesse tipo de reação às adversidades, abordado pela psicologia como estresse positivo (ou eustresse), o indivíduo passa por uma ativação e é impelido a adaptar-se a uma situação interpretada como desafio, gerando como resultantes ânimo, vigor, produtividade e criatividade (Perrewé et al., 2012).

Comparando-se o comportamento das dimensões ao longo dos três agrupamentos evidenciou-se que um tratamento homogêneo, sem a identificação de clusters, ignoraria subgrupos significantes para análise. Das regressões dos três clusters, tem-se fundamentação para verificar se a interação entre o fator consolidado de EGT e a PPO será mais bem explicada como uma relação curvilínea, conforme demonstrado na Tabela 5.

Tabela 5. Resultados das regressões com componente quadrático.

\begin{tabular}{|c|c|c|c|c|}
\hline Etapas & $\boldsymbol{\beta}$ & & $\mathbf{R}^{2}$ & $\Delta \mathrm{F}$ \\
\hline Etapa 1 - Componente linear & & & 0,121 & $12,45 * * *$ \\
\hline EGT & $-0,051$ & $* *$ & & \\
\hline Nível hierárquico & 0,080 & & & \\
\hline Gênero & 0,060 & & & \\
\hline Tempo de serviço & 0,050 & & & \\
\hline Etapa 2 - Inclusão do componente quadrático & & & 0,372 & $818,9 * * *$ \\
\hline EGT $^{2}$ & 0,205 & $* * *$ & & \\
\hline$\Delta \mathrm{R}^{2}$ & 0,251 & & & \\
\hline
\end{tabular}

Nota. Essa tabela apresenta o resultado da regressão hierárquica com a confirmação da Hipótese 4: EGT e PPO apresentam uma relação curvilínea quadrática positiva. Fonte: Dados da Pesquisa. Variável dependente: PPO. *** Significante ao nível de 1\%; ** Significante ao nível de $5 \%$. 
Como se pode ver no modelo não linear, o coeficiente $\beta(-0,051)$ é negativo e significante enquanto $o$ termo quadrático para EGT foi positivo $(\beta=0,205)$ e significante, suportando $\mathbf{H}_{4}$. De fato, o modelo de regressão curvilínea explica uma proporção maior da variância. A Figura 1 ilustra esta associação não linear quadrática positiva (U-shaped form):

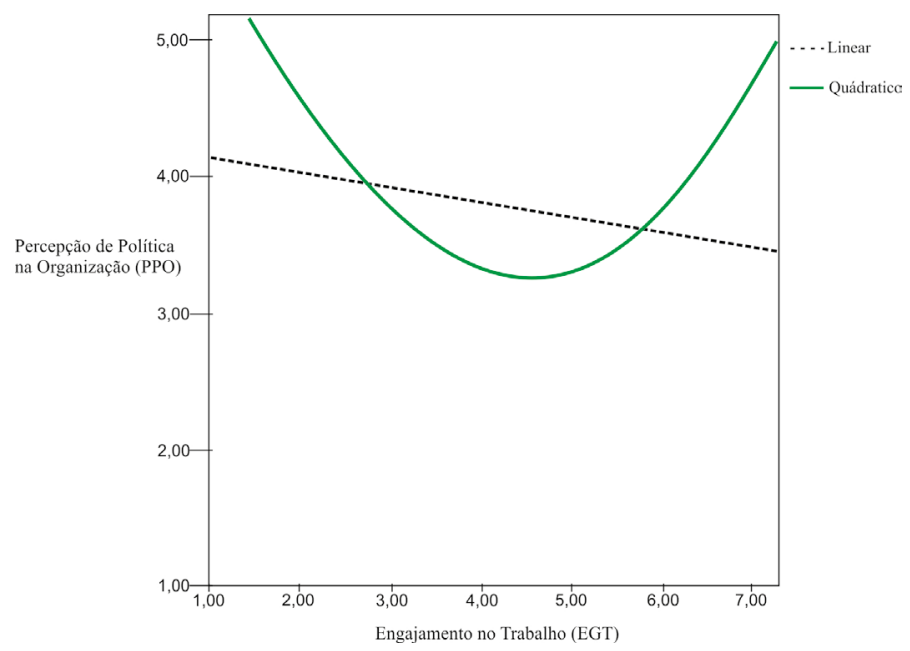

Figura 1. Curva de estimação EGT x PPO (função linear x função quadrática).

Essa figura demonstra a relação não linear entre EGT e PPO. O EGT influencia a redução da PPO na maior parte da observação, porém em níveis elevados passa a contribuir para o aumento da Percepção de Política (trecho ascendente da curva). Fonte: Dados da pesquisa.

Percebe-se que o efeito mitigador do EGT frente a PPO atua na maior parte da observação, porém, como acompanhado na descrição dos clusters, os conceitos são não totalmente antagônicos como poderia sugerir uma forma relacional exclusivamente linear. O comportamento das dimensões nos testes das hipóteses, contribui para fundamentar esta proposição. O trecho ascendente da curva pode ser explicado a partir da presença marcante de política nas organizações brasileiras (De Moraes \& Teixeira, 2017; Torres et al., 2015), nas quais a percepção do fenômeno pode sinalizar um estímulo ao engajamento para os que têm habilidades políticas e almejam crescimento pessoal ou organizacional (Hochwarter et al., 2010; Munyon, Summers, Thompson, \& Ferris, 2015). Em cenários como esse, as atividades políticas podem ser sancionadas socialmente ou almejadas, em função de traços culturais (Chu \& Wood, 2008).

\section{CONCLUSÕES}

Partindo de uma amostra ampla e heterogênea de gestores, este estudo investigou as relações entre os construtos Engajamento no Trabalho e Percepção de Política na Organização. Para um maior aprofundamento das análises os seguintes caminhos foram percorridos: primeiramente identificaram-se grupos com EGT baixo, médio e alto; posteriormente, regressões hierárquicas foram conduzidas em cada um desses grupos para testar o efeito das dimensões constituintes do EGT (Vigor, Dedicação e Absorção) frente à PPO e, por fim, verificou-se junto à amostra total a hipótese de uma relação curvilínea entre os construtos consolidados.

O caminho traçado pelo EGT através de uma associação não linear quadrática positiva com a PPO traz à luz a existência de um ponto de inflexão na relação entre os dois conceitos estudados e sinaliza em que medida os atores começam a ajustar seus papéis organizacionais: primeiramente, nos grupos de gestores com engajamento baixo e médio, o EGT teve uma função mitigadora frente a PPO. Todavia, isto não significa que a política, inerente às organizações, será necessariamente reduzida, mas que na maioria dos casos, com resiliência, envolvimento e concentração é possível experimentar menos esta percepção, normalmente apontada como prejudicial ao desempenho. Posteriormente esse paradigma é quebrado quando se identificou, nos grupos de engajamento elevado, uma associação positiva entre EGT e PPO, flexionando ascendentemente a curva.

Abordando os resultados dos grupos de EGT Baixo e Médio sob a perspectiva do Comportamento Organizacional Positivo, é possível sugerir que fomentar as dimensões do EGT pode propiciar consequências mais efetivas do que tentativas de coibir os comportamentos políticos, pois se trata de um fenômeno natural, sujeito à ambiguidade e variações de ponto de vista. Vale ressaltar que já no nível intermediário ocorre, mesmo que em menor magnitude, uma associação positiva da dedicação com a PPO, sinalizando que, emocionalmente, estes indivíduos já começam a se tornar mais conectados ao jogo político.

Entre os altamente engajados, os componentes emocionais e cognitivos do EGT relacionaram-se de uma forma menos típica à PPO. O terceiro cluster analisado apresentou uma caracterização importante: níveis de engajamento e percepção de política extremamente elevados e uma associação positiva altamente significativa entre dedicação, absorção e política. Tem-se um indício relevante de que gestores emocionalmente e cognitivamente estruturados tendem a aumentar seu engajamento juntamente com a percepção dos 
aspectos estressores de seus ambientes de trabalho, caracterizando que esses profissionais têm um maior entendimento dos meandros organizacionais. Os achados apontam para a capacidade que os gestores com esse perfil têm para reconhecer e até mesmo capitalizar a PPO como uma oportunidade para elaborar estratégias geradoras de benefícios individuais ou organizacionais. Tem-se então uma indicação de que gestores altamente engajados possuem uma carga maior de recursos para reconhecer a inevitabilidade do fenômeno político e seguir em frente.

Outra ponderação importante remete à caracterização do cenário estudado, no qual historicamente há forte presença de comportamentos políticos (Ardichvili et al., 2012; Torres et al., 2015), permitindo sugerir uma aceitação tácita quanto a manobras e formação de alianças não sancionadas, possibilitando a coexistência de escores de EGT e PPO tão elevados.

É relevante salientar que o instrumento de medida utilizado no estudo mediu uma percepção sobre a organização e não a atividade política do entrevistado. Evidentemente o ambiente organizacional deve ser o mais transparente possível, mas a PPO depende de diferentes prismas que envolvem questões culturais e individuais. Assim, as implicações práticas desta pesquisa sugerem políticas voltadas primeiramente ao fortalecimento dos fatores emocionais e cognitivos dos gestores de forma a conectá-los aos sensos de oportunidade e desafio.

Identificados de forma complementar aos objetivos do estudo, alguns indícios merecem

\section{REFERÊNCIAS}

Abbas, M., Raja, U., Darr, W., \& Bouckenooghe, D. (2014). Combined effects of perceived politics and psychological capital on job satisfaction, turnover intentions, and performance. Journal of Management, 40(7), 1813-1830. https://doi. org/10.1177/0149206312455243

Allen, R. W., Porter, L. W., \& Angle, H. L. (2016). Organizational influence processes. New York: Routledge. https://doi. org/10.4324/9781315290614

Angst, R., Benevides-Pereira, A. M. T., \& Porto-Martins, P. C. (2009). UWES manual - português BR. GEPEB-Grupo de Estudos e Pesquisas sobre Estresse e Burnout. Retrieved from https://www.wilmarschaufeli.nl/publications/ Schaufeli/Test\%20Manuals/Test_manual_UWES_Brazil. pdf destaque. Primeiramente verificou-se que, apesar de elevada, a PPO na iniciativa privada foi menor que a do setor público e, de forma inversa, o EGT apresentou escores maiores. Entretanto esta comparação carece de comprovações em desenhos de pesquisa específicos. O outro ponto é que a proximidade dos gestores aos centros de poder impacta no aumento da PPO, sem prejudicar o engajamento, o que reforça os indicativos de que gestores engajados são capazes de instrumentalizar os comportamentos políticos de forma positiva.

O estudo limitou-se a gestores, todos graduados na área de administração e filiados ao Conselho de Classe (CRA), deixando lacunas sobre possíveis resultados com profissionais de outras áreas de formação ou ainda, quanto àqueles que exercem a atividade sem esta formação acadêmica. A remuneração não foi considerada como variável de controle, permitindo sugerir pesquisas que ponderem a influência financeira nos dois conceitos aqui abordados. Futuras investigações sobre a relação entre EGT e PPO com comparações transnacionais poderão contribuir para entender em que medida os desafios e similaridades entre diferentes economias e contextos culturais estendem-se às questões comportamentais aqui analisadas. Espera-se que esse estudo incite novas discussões sobre política organizacional e suas relações com outras medidas de comportamento organizacional, de modo a quebrar o tabu sobre este assunto inerente ao cotidiano das empresas.

Ardichvili, A., Jondle, D., Kowske, B., Cornachione, E., Li, J., \& Thakadipuram, T. (2012). Ethical cultures in large business organizations in Brazil, Russia, India, and China. Journal of Business Ethics, 105(4), 415-428. https://doi.org/10.1007/s10551-011-0976-9

Atinc, G., Darrat, M., Fuller, B., \& Parker, B. W. (2010). Perceptions of organizational politics: A meta-analysis of theoretical antecedents. Journal of Managerial Issues, 22(4), 494-513. Retrieved from http://www.jstor.org/stable/25822527

Bakker, A. B., Albrecht, S. L., \& Leiter, M. P. (2011). Work engagement: Further reflections on the state of play. European Journal of Work and Organizational Psychology, 20(1), 74-88. https://doi.org/10.1080/1359432X.2010.546711 
Bakker, A. B., \& Demerouti, E. (2008). Towards a model of work engagement. Career Development International, 13(3), 209-223. https://doi. org/10.1108/13620430810870476

Bakker, A. B., \& Demerouti, E. (2017). Job demands-resources theory: Taking stock and looking forward. Journal of Occupational Health Psychology, 22(3), 273-285. https://doi.org/10.1037/ocp0000056

Bedi, A., \& Schat, A. C. H. (2013). Perceptions of organizational politics: A meta-analysis of its attitudinal, health, and behavioural consequences. Canadian Psychology/ Psychologie Canadienne, 54(4), 246-259. http://dx.doi. org/10.1037/a0034549

Brasil. (2017). Ministério da Educação. Instituto Federal do Espírito Santo-CEP Ifes. Retrieved from https://www. ifes.edu.br/conselhos-comissoes/comite-de-etica-empesquisa-do-ifes

Brass, D. J. (2017). Intraorganizational power and dependence. In J. A. C. Baum (Ed.), The Blackwell companion to organizations (Chap. 6, pp. 138-157). Oxford: Blackwell.

Buchanan, D. A. (2008). You stab my back, I'll stab yours: Management experience and perceptions of organization political behaviour. British Journal of Management, 19(1), 49-64. https://doi.org/10.1111/ j.1467-8551.2007.00533.x

Chang, C.-H., Rosen, C. C., \& Levy, P. E. (2009). The relationship between perceptions of organizational politics and employee attitudes, strain, and behavior: A metaanalytic examination. Academy of Management Journal, 52(4), 779-801. https://doi.org/10.5465/ amj.2009.43670894

Chatterjee, S., \& Hadi, A. S. (2015). Regression analysis by example. Hoboken, NJ: John Wiley \& Sons.

Christian, M. S., Garza, A. S., \& Slaughter, J. E. (2011). Work engagement: A quantitative review and test of its relations with task and contextual performance. Personnel Psychology, 64(1), 89-136. https://doi. org/10.1111/j.1744-6570.2010.01203.x

Chu, R. A., \& Wood, T. Jr. (2008). Cultura organizacional brasileira pós-globalização: Global ou local? Revista de Administração Pública, 42(5), 969-994. Retrieved from http://bibliotecadigital.fgv.br/ojs/index.php/rap/ article/view/6661

Cohen, P., West, S. G., \& Aiken, L. S. (2014). Applied multiple regression/correlation analysis for the behavioral sciences. New York: Psychology Press.

Crawford, E. R., LePine, J. A., \& Rich, B. L. (2010). Linking job demands and resources to employee engagement and burnout: A theoretical extension and meta-analytic test. Journal of Applied Psychology, 95(5), 834-848. http://dx.doi.org/10.1037/a0019364

De Moraes, R. M. (2017). Escala de percepção de política na organização: Adaptação e validação para o contexto brasileiro. Revista Eletrônica de Ciência Administrativa, 16(3), 197-212. https://doi.org/10.21529/ RECADM.2017012
De Moraes, R. M., \& Teixeira, A. J. C. (2017). When engagement meets politics: Analysis of a Brazilian public institution. Public Organization Review, 17(4), 495508. https://doi.org/10.1007/s11115-016-0353-3

Eldor, L. (2017). Looking on the bright side: The positive role of organisational politics in the relationship between employee engagement and performance at work. Applied Psychology, 66(2), 233-259. https://doi. org/10.1111/apps.12090

Fedor, D., Maslyn, J., Farmer, S., \& Bettenhausen, K. (2008). The contribution of positive politics to the prediction of employee reactions. Journal of Applied Social Psychology, 38(1), 76-96. https://doi.org/10.1111/ j.1559-1816.2008.00297.x

Ferris, G. R., Frink, D. D., Bhawuk, D. P. S., Zhou, J., \& Gilmore, D. C. (1996). Reactions of diverse groups to politics in the workplace. Journal of Management, 22(1), 23-44. https://doi.org/10.1177/014920639602200102

Gagné, M. (Ed.). (2014). The Oxford handbook of work engagement, motivation, and self-determination theory. New York: Oxford Library of Psychology.

Garson, G. (2014). Cluster analysis: 2014 edition (statistical associates blue book series 24). Asheboro, NC: Statistical Associates Publishing.

Hair, J. F., Black, W. C., Babin, B. J., Anderson, R. E., \& Tatham, R. L. (2006). Multivariate data analysis (vol. 6). Upper Saddle River: Pearson Prentice Hall

Hochwarter, W. A., Ferris, G. R., Laird, M. D., Treadway, D. C., \& Coleman Gallagher, V. (2010). Nonlinear politics perceptions-work outcomes relationships: A three-study, five-sample investigation. Journal of Management, 36(3), 740-763. https://doi. org/10.1177/0149206308324065

Hope, O. (2010). The politics of middle management sensemaking and sensegiving. Journal of Change Management, 10(2), 195-215. https://doi. org/10.1080/14697011003795669

Jackson, M., \& Grace, D. (2018). Machiavelliana: The living machiavelli in modern mythologies. Boston: Brill.

Kacmar, K. M., Bachrach, D. G., Harris, K. J., \& Zivnuska, S. (2011). Fostering good citizenship through ethical leadership: Exploring the moderating role of gender and organizational politics. Journal of Applied Psychology, 96(3), 633-642. http://dx.doi.org/10.1037/a0021872

Kacmar, K. M., Bozeman, D. P., Carlson, D. S., \& Anthony, W. P. (1999). An examination of the perceptions of organizational politics model: Replication and extension. Human Relations, 52(3), 383-416. https:// doi.org/10.1023/A:1016949222512

Kane-Frieder, R. E., Hochwarter, W. A., \& Ferris, G. R. (2014). Terms of engagement: Political boundaries of work engagement - work outcomes relationships. Human Relations, 67(3), 357-382. https://doi. org/10.1177/0018726713495068

Kimura, T. (2015). A review of political skill: Current research trend and directions for future research. International Journal of Management Reviews, 17(3), 312-332. https://doi.org/10.1111/ijmr.12041 
Kreutzer, M., Walter, J., \& Cardinal, L. B. (2015). Organizational control as antidote to politics in the pursuit of strategic initiatives. Strategic Management Journal, 36(9), 13171337. https://doi.org/10.1002/smj.2306

Kaufman, R. L. (2013). Heteroskedasticity in regression: Detection and correction. London: Sage Publications.

LePine, J. A., Podsakoff, N. P., \& LePine, M. A. (2005). A metaanalytic test of the challenge stressor-hindrance stressor framework: An explanation for inconsistent relationships among stressors and performance. Academy of Management Journal, 48(5), 764-775. https://doi.org/10.5465/amj.2005.18803921

Lepisto, D. A., \& Pratt, M. G (2012). Politics in perspectives: On the theoretical challenges and opportunities in studying organizational politics. In G. R. Ferris, \& D. C. Treadway (Eds.), Politics in organizations (Chap. 3, pp. 101-132). New York: Routledge.

Lu, C.-Q., Wang, H.-J., Lu, J.-J., Du, D.-Y., \& Bakker, A. B. (2014). Does work engagement increase person-job fit? The role of job crafting and job insecurity. Journal of Vocational Behavior, 84(2), 142-152. https://doi. org/10.1016/j.jvb.2013.12.004

Luthans, F., Luthans, B. C., \& Luthans, K. W. (2015). Organizational behavior: An evidence-based approach. Charlotte: Information Age Publishing.

Madison, D. L., Allen, R. W., Porter, L. W., Renwick, P. A., \& Mayes, B. T. (1980). Organizational politics: An exploration of managers' perceptions. Human Relations, 33(2), 79100. https://doi.org/10.1177/001872678003300201

Mansur, J. A., \& Sobral, F. (2011). Política na terra do 'jeitinho’: Consequências dos comportamentos políticos em organizações no Brasil. Revista de Administração Mackenzie, 12(6), pp. 165-191. Retrieved from http:// www.redalyc.org/articulo.oa?id=195421147008

Meisler, G., \& Vigoda-Gadot, E. (2014). Perceived organizational politics, emotional intelligence and work outcomes: Empirical exploration of direct and indirect effects. Personnel Review, 43(1), 116-135. https://doi. org/10.1108/PR-02-2012-0040

Miller, B. K., Rutherford, M. A., \& Kolodinsky, R. W. (2008). Perceptions of organizational politics: A meta-analysis of outcomes. Journal of Business and Psychology, 22(3), 209-222. https://doi.org/10.1007/s10869-0089061-5

Mintzberg, H. (1985). The organization as political arena. Journal of Management Studies, 22(2), 133-154. https://doi.org/10.1111/j.1467-6486.1985.tb00069.x

Miranda, S. (2009). O tabu do poder e da política nos estudos organizacionais. Revista de Administração da UFSM, 2(1), 10-20. Retrieved from https://periodicos.ufsm. $\mathrm{br} /$ reaufsm/article/view/1262

Munyon, T. P., Summers, J. K., Thompson, K. M., \& Ferris, G. R. (2015). Political skill and work outcomes: A theoretical extension, meta-analytic investigation, and agenda for the future. Personnel Psychology, 68(1), 143-184. https://doi.org/10.1111/peps.12066
Nishii, L. H., \& Wright, P. M. (2008). Variability within organizations: Implications for strategic human resources management. In D. B. Smith (Ed.), LEA's organization and management series. The people make the place: Dynamic linkages between individuals and organizations (pp. 225-248). New York: Taylor \& Francis Group/Lawrence Erlbaum Associates.

Parker, C. P., Dipboye, R. L., \& Jackson, S. L. (1995). Perceptions of organizational politics: An investigation of antecedents and consequences. Journal of Management, 21(5), 891912. https://doi.org/10.1177/014920639502100505

Perrewé, P. L., Rosen, C. C., \& Maslach, C. (2012). Organizational politics and stress: The development of a process model. In G. R. Ferris, \& D. C. Treadway (Eds.), Politics in organizations: Theory and research considerations (Chap. 8, pp. 247-290). New York: Routledge.

Reijseger, G., Peeters, M. C., Taris, T. W., \& Schaufeli, W. B. (2017). From motivation to activation: Why engaged workers are better performers. Journal of Business and Psychology, 32(2), 117-130. https://doi.org/10.1007/ s10869-016-9435-z

Rich, B. L., Lepine, J. A., \& Crawford, E. R. (2010). Job engagement: Antecedents and effects on job performance. Academy of Management Journal, 53(3), 617-635. https://doi. org/10.5465/amj.2010.51468988

Russo, R. F. S. M., Rodrigues, F., Russo, R., \& Yu, A. S. O. (2018). Comportamento político nas organizações: Mecanismos de intervenção. Revista Eletrônica de Ciência Administrativa, 17(3), 295-315. https://doi. org/10.21529/RECADM.2018012

Sarstedt, M., \& Mooi, E. (2014). A concise guide to market research. New York: Springer.

Schaufeli, W. B. (2012). Work engagement: What do we know and where do we go? Romanian Journal of Applied Psychology, 14(1), 3-10. Retrieved from http://www. rjap.psihologietm.ro/Download/rjap141_1.pdf

Schaufeli, W. B., Bakker, A. B., \& Salanova, M. (2006). The measurement of work engagement with a short questionnaire: A cross-national study. Educational and Psychological Measurement, 66(4), 701-716. https:// doi.org/10.1177/0013164405282471

Schaufeli, W. B., Salanova, M., González-Romá, V., \& Bakker, A. B. (2002). The measurement of engagement and burnout: A two sample confirmatory factor analytic approach. Journal of Happiness studies, 3(1), 71-92. https://doi.org/10.1023/A:1015630930326

Seppälä, P., Mauno, S., Feldt, T., Hakanen, J., Kinnunen, U., Tolvanen, A., \& Schaufeli, W. (2009). The construct validity of the Utrecht Work Engagement Scale: Multisample and longitudinal evidence. Journal of Happiness Studies, 10(4), 459. https://doi. org/10.1007/s10902-008-9100-y

Smollan, R. K. (2014). Control and the emotional rollercoaster of organizational change. International Journal of Organizational Analysis, 22(3), 399-419. https://doi. org/10.1108/IJOA-08-2012-0609 
Torres, C. V., Alfinito, S., Galvão, C. A. D. S. P., \& Tse, B. C. Y. (2015). Brazilian jeitinho versus Chinese guanxi: Investigating their informal influence on international business. RAM. Revista de Administração Mackenzie, 16(4), 77-99. http://dx.doi.org/10.1590/167869712015/administracao.v16n4p77-99

\section{Autores}

\section{Romulo Matos de Moraes*}

Rod Br 482, Km 47 s/n, 29520-000, Alegre, ES, Brasil.

E-mail: romulomoraes@hotmail.com; romuloifes@gmail.com

ㄴ) https://orcid.org/0000-0003-2357-0869

\section{Aridelmo José Campanharo Teixeira}

Av. Fernando Ferrari, no 1358, Boa Vista, 29075-505, Vitória, ES, Brasil.

E-mail: aridelmo@fucape.br

(ㄱ) https://orcid.org/0000-0002-4909-1025

* Autor Correspondente

\section{Contribuições dos Autores}

$1^{\circ}$ autor: Elaboração do problema de pesquisa; revisão de literatura; coleta dos dados; definição do método; análise dos dados; interpretação dos resultados; redação do manuscrito.

$2^{\circ}$ autor: Elaboração do problema de pesquisa; indicação da teoria; validação da análise dos dados; revisão crítica do manuscrito.

\section{Financiamento}

Os autores informaram que não houve apoio financeiro para a pesquisa neste artigo.
Vigoda, E. (2001). Reactions to organizational politics: A cross-cultural examination in Israel and Britain. Human Relations, 54(11), 1483-1518. https://doi. org/10.1177/00187267015411004

Warr, P. B. (2011). Work, happiness, and unhappiness. London: Psychology Press.

\section{Conflito de Interesses}

Os autores informaram que não há conflito de interesses.

\section{Direitos Autorais}

A RAC detém os direitos autorais deste conteúdo.

\section{Verificação de Plágio}

A RAC mantém a prática de submeter todos os documentos aprovados para publicação à verificação de plágio, mediante o emprego de ferramentas específicas, e.g.: iThenticate.

\section{Método de Revisão por Pares}

Este conteúdo foi avaliado utilizando o processo de revisão por pares duplo-cego (double-blind peer-review). A divulgação das informações dos pareceristas constantes na primeira página é feita somente após a conclusão do processo avaliativo, e com o consentimento voluntário dos respectivos pareceristas.

\section{Disponibilidade dos Dados}

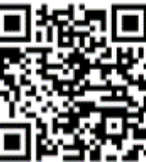

Todos os dados e materiais foram disponibilizados publicamente por meio da plataforma Mendeley e podem ser acessados em: De Moraes, Romulo Matos; Teixeira, Aridelmo José Campanharo (2020). Replication Data for: "Full Data used for: Managers, Engagement and Political Behaviors: a nonlinear relationship. Published by RAC-Revista de Administração Contemporânea (Journal of Contemporary Administration)", 24(3), 2020. [Data set]. Journal of Contemporary Administration (RAC). Mendeley. http://doi. org/10.17632/syrw7xgynt.1 